\title{
A theoretical and experimental analysis of modulated laser fields and power spectra
}

Olesen, Henning; Jacobsen, G.

Published in:

I E E E Journal of Quantum Electronics

Link to article, DOI:

10.1109/JQE.1982.1071490

Publication date:

1982

Document Version

Publisher's PDF, also known as Version of record

Link back to DTU Orbit

Citation (APA):

Olesen, H., \& Jacobsen, G. (1982). A theoretical and experimental analysis of modulated laser fields and power spectra. I E E E Journal of Quantum Electronics, 18(12), 2069-2080. https://doi.org/10.1109/JQE.1982.1071490

\section{General rights}

Copyright and moral rights for the publications made accessible in the public portal are retained by the authors and/or other copyright owners and it is a condition of accessing publications that users recognise and abide by the legal requirements associated with these rights.

- Users may download and print one copy of any publication from the public portal for the purpose of private study or research.

- You may not further distribute the material or use it for any profit-making activity or commercial gain

- You may freely distribute the URL identifying the publication in the public portal

If you believe that this document breaches copyright please contact us providing details, and we will remove access to the work immediately and investigate your claim. 


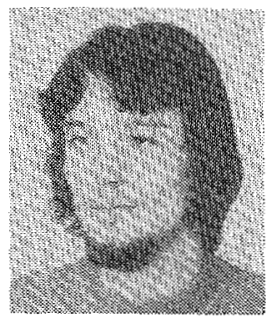

Joachim Heppner was born in Hannover, West Germany, on October 20, 1950. He received the diploma in physics from the Technische Universität, Hannover, West Germany, in 1976. From 1976 to 1980 he was with the Physikalische-Technische, Bundesanstalt Braunschweig, West Germany, where his research was on $\mathrm{CW}$ FIR lasers. Since March 1980 he has been with the Universität Stuttgart, Stuttgart, West Germany, where his research includes pulsed FIR laser in connection with plasma diagnostics.

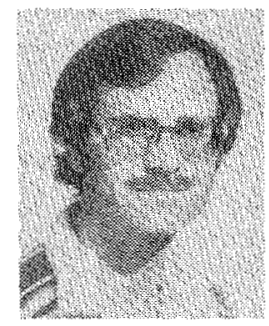

Frank B. Foote (M'80) was born on January 10,1947 . He received the B.S. degree in physics from the California State University, Long Beach, in 1975, and the M.S.E.E. degree in quantum electronics from the University of Southern California, Los Angeles, in 1980.

$\mathrm{He}$ is currently a member of the Technical $S$ taff employed by the Aerospace Corporation, El Segundo, CA. His areas of interest are far infrared/near millimeter wave lasers, millimeter wave radiometer and imaging, and lidar and digital electronics.

\title{
A Theoretical and Experimental Analysis of Modulated Laser Fields and Power Spectra
}

\author{
HENNING OLESEN AND GUNNAR JACOBSEN
}

\begin{abstract}
A general theoretical description of modulated laser fields and power spectra for a current modulated single-mode laser is derived, taking into account both the intensity and frequency modulation (IM and FM) of the emitted light. The theory relies on an explicit knowledge of the modulus as well as the phase of the current-to-frequency modulation transfer function for the laser. Numerical examples are presented for sinusoidal, sawtooth, and square wave modulation considering broad-band and narrow-band FM cases with various amounts of IM. The IM causes a significant distortion of the pure FM spectrum, strongly dependent on the modulus and the phase of the current-tofrequency modulation transfer function. In general, it causes the FM spectrum to become asymmetrical with a change of the relative sideband level. The theoretical results have been confirmed experimentally by Fabry-Perot interferometer measurements on a temperature stabilized CSP injection laser. In the interpretation of the measurement results, the detailed characteristics of the interferometer, and the detection system are taken into account. The measurements include narrowband and broad-band sinusoidal modulation as well as broad-band sawtooth and square wave modulation.
\end{abstract}

\section{INTRODUCTION}

$\mathrm{C}$ OHERENT optical transmission systems, using angular modulation of a highly coherent injection laser, look very promising for future long-distance, high-capacity transmission [1]-[3]. Several methods for achieving frequency modulation (FM) or phase modulation (PM) of the emitted light have been investigated and, in particular, direct optical FM by injection current modulation has received considerable attention [4]-[6] .

It is important to realize that direct current modulation always results in combined intensity and frequency modulation (IM and FM). For coherent applications the IM is an undesired

Manuscript received April 18, 1982; revised July 16, 1982.

The authors are with the Electromagnetics Institute, Technical University of Denmark, DK-2800 Lyngby, Denmark. effect, but since the frequency deviation per unit current is in the range from $0.1-3.5 \mathrm{GHz} / \mathrm{mA}$ [6] the spurious IM will often be small. If the IM cannot be tolerated, it may be reduced by the use of injection locked repeaters [7], [8] .

In conventional systems, however, large signal IM is often employed, and in this case the FM is the unwanted part. By careful design of the system the FM does not cause any trouble, but it may have a serious influence on system performance if interference effects such as modal noise [9], [10] and polarization noise [11], [12] are not eliminated.

For both applications, a proper characterization of the injection laser and an understanding of the IM-FM interaction is important. Earlier papers have dealt with the physical explanation of FM through thermal and carrier effects [13]-[15] and with the measurement of modulus [6] and phase [16] of the current-to-frequency modulation transfer function (CFMTF). Examples of frequency variation under different modulation conditions have also been presented [12], [14], together with modulated power spectra [3], [5] , [6] , [14] .

In this paper we present a general theory for derivation of the total (IM + FM) modulation function for the laser field with arbitrary current modulation, based on the knowledge of the transfer function (CF-MTF) mentioned above. In particular, we shall consider periodic signals such as sinusoidal, sawtooth, and square wave modulation. Furthermore, the modulated power spectrum will be derived and numerical examples, as well as experimental results, will be presented and compared. A thorough theoretical analysis is given in Section II, while Sections III and IV present results of a numerical and experimental study of modulated laser spectra. An analysis of some of the practical problems in connection with Fabry-Perot interferometer (FPI) measurements is given in the Appendix. 


\section{THEORY}

A. Derivation of the Modulation Function for the Electrical Field

In the following we shall derive the complex modulation function $m(t)$ for arbitrary current modulation, defined as the ratio between the modulated laser field and the unmodulated laser field.

In order to account for the finite linewidth (partial coherence) of the source the unmodulated laser field is written as [17]

$$
E_{o}(t)=r(t) e^{j 2 \pi f_{o} t} \text {. }
$$

The function $r(t)$ is a (complex) random stationary process with the property that

$$
\left\langle r(t+\tau) r^{*}(t)\right\rangle=R_{o}(\tau)
$$

The brackets and the asterix denote ensemble average and complex conjugate, respectively, $R_{o}(\tau)$ is the (low frequency) autocorrelation function of the unmodulated laser and $f_{o}$ is the optical carrier frequency.

By standard relations we get

$$
R_{o}(\tau)=\int_{-\infty}^{\infty} S_{o}(f) e^{j 2 \pi f \tau} d f
$$

which connects $r(t)$ to physically measurable characteristics. $S_{o}(f)$ is the power spectrum of the unmodulated laser shifted to zero center frequency in the following named the spectral line shape of the laser.

The modulated laser field can now be written as

$$
E(t)=m(t) r(t) e^{i 2 \pi f_{O} t}
$$

and the effect of the modulation can be conveniently separated from that due to the partial coherence. The modulation function will include both intensity modulation (IM) and frequency modulation (FM).

For simplicity, we will assume that the injection current (bias + modulation) is always greater than or equal to the threshold current $I_{\text {th }}$. Transient IM effects are neglected, i.e., it is assumed that the output light intensity always follows the imposed current modulation exactly, without any time delay. If necessary (at high frequencies) the theory may be extended to include an additional complex current-to-intensity modulation transfer function.

The laser current is represented by

$$
I(t)=I_{o}+I_{m} \cdot f(t)
$$

where $I_{o}$ is the dc bias current, $I_{m}$ is the modulation current, and $f(t)$ is a function specifying the current modulation, with a minimum value of -1 .

This representation is mainly suited for symmetric modulation without $\mathrm{dc}$ content, but in case of asymmetry the dc current may be included in $I_{O}$ and (5) remains valid. Assuming a linear light current characteristic of the laser, the output power becomes

$$
\begin{aligned}
P(t) & =\frac{d P}{d I} \cdot\left[I(t)-I_{\mathrm{th}}\right] \\
& =P_{o}+P_{m} \cdot f(t)
\end{aligned}
$$

where $P_{o}=d P / d I \cdot\left(I_{o}-I_{\mathrm{th}}\right)$ and $P_{m}=d P / d I \cdot I_{m}$ are the unmodulated power and the modulation power, respectively.

Both equations may be normalized yielding a normalized output intensity and current

$$
\left.\begin{array}{l}
p(t) \\
i(t)
\end{array}\right\}=1+m f(t)
$$

where $m$ is an IM modulation index given as

$$
m=\frac{I_{m}}{I_{o}-I_{\mathrm{th}}}=\frac{P_{m}}{P_{o}} .
$$

Again, the concept of an IM index is most meaningful for symmetric modulation without any dc content.

As the output intensity is known to be proportional to the squared modulus of the electric field we find as the first important result that

$$
|m(t)|=\sqrt{1+m f(t)},
$$

i.e., the modulus of the modulation function is the square root of the normalized modulation current.

It is a characteristic feature of the direct optical FM that it is unavoidably accompanied by intensity modulation (and vice versa). Various steps may be taken to remove either part, e.g., use of injection locked repeaters [7] as limiters and frequency stabilization by the use of an external cavity [18] .

Next, the optical frequency modulation is considered. Here it is assumed that the CF-MTF of the laser is known, either from theoretical investigations or from measurements of the modulus and phase [6], [16]. The complex CF-MTF is written as

$$
H_{f}\left(I_{o}, f\right)=\left|H_{f}\left(I_{o}, f\right)\right| e^{j \phi_{f}\left(I_{O}, f\right)}
$$

typically measured in units of $\mathrm{GHz} / \mathrm{mA}$. It seems reasonable to assume that

$$
H_{f}\left(I_{o},-f\right)=H_{f}^{*}\left(I_{o}, f\right) .
$$

Both modulus and phase are functions of the bias current $I_{o}$, especially at higher frequencies, where the carrier effect is dominating. This may introduce nonlinearities in the case of large-signal modulation, which are not included in the present model.

From (10) and (11) the corresponding temporal impulse response may (in principle) be calculated by Fourier transformation

$$
h_{f}\left(I_{o}, t\right) \triangleq \int_{-\infty}^{\infty} H_{f}\left(I_{o}, f\right) e^{j 2 \pi f t} d f
$$

and the optical frequency modulation due to $f(t)$ is then obtained by convolution (" $\oplus$ ")

$$
\nu(t)=-I_{m} f(t) \oplus h_{f}\left(I_{o}, t\right) .
$$

Here the negative sign accounts for the fact that the frequency and current modulation are in counterphase at low frequency [4], where the thermal effect is dominating. The phase of $H_{f}$ is accordingly assumed to go to zero at low frequencies.

The temporal phase variation due to $f(t)$ is found by integra- 
tion of (13)

$$
\begin{aligned}
\theta(t) & =2 \pi \int \nu\left(t^{\prime}\right) d t^{\prime} \\
& =-2 \pi I_{m} \int\left[f\left(t^{\prime}\right) \oplus h_{f}\left(I_{o}, t^{\prime}\right)\right] d t^{\prime}
\end{aligned}
$$

Finally, combining (9) and (14) yields the general expression for the complex modulation function

$m(t)=\sqrt{1+m f(t)} \exp \left\{-j 2 \pi I_{m} \cdot \int\left[f\left(t^{\prime}\right) \oplus h_{f}\left(I_{o}, t^{\prime}\right)\right] d t^{\prime}\right\}$

which includes both IM and FM. The steps involved in the construction of $m(t)$ from the dc bias and modulation current are illustrated schematically in Fig. 1. The nonuniform frequency response $H_{f}\left(I_{o}, f\right)$ is modeled as a linear network possessing the same amplitude transfer function.

As a simple example illustrating the theory let us consider the case of sinusoidal modulation. The function specifying the current modulation is now given as

$$
f(t)=\sin \left(2 \pi f_{m} t\right)
$$

where $f_{m}$ is the modulation frequency.

The temporal frequency variation (13) becomes

$$
\begin{aligned}
\nu(t) & =-I_{m} \sin \left(2 \pi f_{m} t\right) \oplus h_{f}\left(I_{o}, t\right) \\
& =-\Delta f \cdot \sin \left\{2 \pi f_{m} t+\phi_{f}\left(I_{o}, f_{m}\right)\right\}
\end{aligned}
$$

where

$$
\Delta f=I_{m} \cdot\left|H_{f}\left(I_{o}, f_{m}\right)\right|
$$

is the peak frequency deviation due to the modulation.

Inserting (17) in (14) and integrating we get

$$
\begin{aligned}
m(t)= & \sqrt{1+m \sin \left(2 \pi f_{m} t\right)} \\
& \cdot \exp \left\{j \beta \cos \left[2 \pi f_{m} t+\phi_{f}\left(I_{o}, f_{m}\right)\right]\right\}
\end{aligned}
$$

where $\beta$ is the usual FM index

$$
\beta=\frac{\Delta f}{f_{m}} \text {. }
$$

This case has been analyzed in an earlier paper by the authors [16]. The argument of the CF-MTF enters as a phase delay between intensity and frequency modulation, while the modulus allows a determination of the actual FM index. In case of incoherent detection the (normalized) detected intensity is

$$
i_{d}(t)=1+m \sin \left(2 \pi f_{m} t\right)
$$

as desired.

For arbitrary periodic modulation a similar procedure can be used to determine the modulation function $m(t)$. In this case the phase modulation is obtained by adding the contributions from each Fourier component given as above.

In both cases the knowledge of $f(t)$ and $H_{f}$ is sufficient for the calculation of $m(t)$, i.e., a Fourier transformation of $H_{f}$ is not required. This is obviously convenient as it saves a compu-

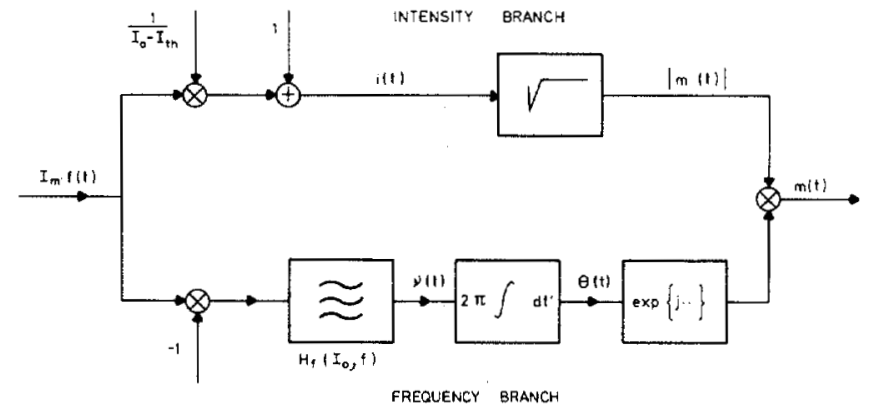

Fig. 1. Schematic diagram illustrating the construction of the modulation function $m(t)(15)$.

tational step and relates $m(t)$ more directly to measurable quantities.

\section{B. Derivation of the Modulated Laser Spectrum}

The modulated power spectrum is an important characteristic of the laser, both for coherent and incoherent applications. In coherent systems the FM index is a measure of the necessary bandwidth of the transmission system, and for incoherent analog transmission, the modulated spectrum will be indicative of the harmonic distortion components arising from, for example, modal distortion [10].

In this analysis we will concentrate on periodic modulation, but an extension to the general case is straightforward. If $f(t)$ is assumed to be periodic, then $m(t)$ will be periodic with the same period $T$. It may, therefore, be expanded in a Fourier series

$$
m(t)=\sum_{n=-\infty}^{\infty} \gamma_{n} e^{j 2 \pi n f_{m} t}, \quad f_{m}=\frac{1}{T}
$$

with

$$
\gamma_{n}=\int_{0}^{1} m\left(\frac{\alpha}{f_{m}}\right) e^{-j 2 \pi n \alpha} d \alpha .
$$

The modulated laser field (4) is neither periodic nor stationary, so in order to obtain a time-independent autocorrelation function, both a time and ensemble average is required [19]

$$
R(\tau)=\frac{1}{T} \int_{0}^{T}\left\langle E(t+\tau) E^{*}(t)\right\rangle d t .
$$

The ensemble average affects only $r(t)$ and the result is

$$
R(\tau)=R_{m}(\tau) \cdot R_{o}(\tau) \cdot e^{j 2 \pi f_{o} \tau}
$$

where $R_{o}(\tau)$ is given by (2) and

$$
R_{m}(\tau)=\sum_{n=-\infty}^{\infty}\left|\gamma_{n}\right|^{2} e^{j 2 \pi n f_{m} \tau}
$$

is the autocorrelation function of the modulation.

From (25) and (26) the modulated laser spectrum is obtained by Fourier transformation

$$
\begin{aligned}
S(f) & =S_{m}(f) \oplus S_{o}(f) \oplus \delta\left(f-f_{o}\right) \\
& =S_{m}(f) \oplus S_{o}\left(f-f_{o}\right) \\
& =\sum_{n=-\infty}^{\infty}\left|\gamma_{n}\right|^{2} S_{o}\left(f-f_{o}-n f_{m}\right) .
\end{aligned}
$$


This expression shows that the modulated laser spectrum is obtained by translation of $S_{m}(f)$ to a center frequency of $f_{o}$ followed by a convolution of each sideband with the spectral line shape of the unmodulated laser. Depending on the relation between the modulation frequency and the spectral linewidth, the spectrum will consist of separated or overlapping versions of $S_{o}(f)$. The relative height of the individual sidebands is determined only by the modulation and the influence of various modulation parameters may, therefore, be studied by analyzing only $S_{m}(f)$ as the translation and convolution with $S_{o}(f)$ are easily performed when necessary.

In the high frequency region, where $f_{m}$ is much greater than the spectral linewidth, the spectrum may be correctly recorded by an FPI analysis, apart from a possible line broadening due to the finite FPI resolution (see the Appendix). In the low frequency region the FM index is always very high, and in this case, the spectrum $S_{m}(f)$ approaches a limit distribution [20] given by (A23)

$$
S_{\text {lim }}(f)=|m(t(f))|^{2} \cdot w(f)
$$

Here $w(\nu)$ is the probability density of the instantaneous frequency deviation $\nu(t)(13)$ and $|m(t(\nu))|^{2}$ is equal to the normalized intensity (7) at the time where $\nu(t)$ equals $\nu$. A correct FPI measurement in this case is more complicated (see the Appendix).

Usually the influence of IM and FM on the modulated laser spectrum are difficult to separate. The expression for $m(t)$ (15) implies a multiplication of the square-root IM term and the exponential FM term. This means that the complex amplitude spectrum of $m(t)$ is obtained by convolution of the amplitude spectra for the IM and FM terms. In order to get from the amplitude spectrum to the power spectrum, we then take the squared modulus of the complex amplitude of each sideband, as indicated by (27). However, in many cases the number of sidebands due to the frequency modulation will largely exceed the number due to the intensity modulation. This is illustrated in the next section for the case of sinusoidal modulation.

The model presented in this and the previous section is adequate for most purposes and provides a good understanding of the IM-FM interaction. However, there are effects which cannot be treated by the simple approach, such as, e.g., the line broadening and multimode operation observed for highfrequency modulation. This implies a coupling between the modulation and the source properties which causes deviations from the assumed stationarity. These effects seem to require further investigations in order to obtain a thorough understanding.

\section{Numerical ExAmples}

In this section results of a numerical investigation of modulated laser spectra will be presented. The influence of the IM index $m$, the FM index $\beta$, and the phase delay $\phi$ of the transfer function $H_{f}$ will be demonstrated. The types of modulation considered are sinusoidal, sawtooth, and square wave modulation.

\section{A. Sinusoidal Modulation}

The plots, which are presented in the following, may be scaled arbitrarily with the modulation frequency, as the independent variable is the number of the actual Fourier compo- nent, i.e., the harmonics number, and only the spectrum $S_{m}(f)$ is considered. This has been justified earlier in this paper, since the field spectrum may be obtained from $S_{m}(f)$ by a parallel shift and a convolution with $S_{o}(f)$.

The interesting parameters to vary are therefore $m, \beta$, and $\phi=\phi_{f}\left(I_{o}, f_{m}\right)$ and the following examples will illustrate their influence. The modulation function $m(t)$ is given by (19). At first, we consider the IM term

$$
m_{I}(t)=\sqrt{1+m \sin \left(2 \pi f_{m} t\right)}
$$

Fig. 2 shows the time variation of $m_{I}(t)$ over one period with $m$ as parameter. For small $m$ values $m_{I}(t)$ may be approximated with a sine wave

$$
m_{I}(t) \simeq 1+\frac{m}{2} \sin \left(2 \pi f_{m} t\right)
$$

while for $m$ approaching 1 the function deviates substantially from a sine wave.

In Fig. 3 we have plotted in logarithmic scale the power spectrum of $m_{I}(t)(29)$ also with $m$ as parameter. It is seen that for small $m$ values only few harmonics are significant, whereas the spectrum broadens strongly as $m$ approaches 1 . This is, of course, to be expected from Fig. 2.

A plot in linear scale, Fig. 4, shows the power level of the carrier and first sideband as a function of $m$. The carrier decreases from 1 to about 0.7 as $m$ goes from 0 to 1 while the first sideband is only just visible in this linear plot. However, although the spectrum of $m_{I}(t)$ is narrow with only few significant components, it has a strong influence on the combined IM-FM spectrum $S_{m}(f)$ as shown in the following.

When analyzing the spectrum $S_{m}(f)$ with $m(t)$ given by (19), one must keep in mind that the parameters $m, \beta, f_{m}$, and $\phi$ cannot be varied independently. The modulus and phase of the transfer function $H_{f}\left(I_{o}, f\right)$ behave as shown in Fig. 5(a) and (b), taken from [16]. At low frequencies $\left|H_{f}\right|$ is large so that $m$ should be close to zero, $\beta$ much greater than 1 and in addition $\phi$ close to zero. Between $10 \mathrm{MHz}$ and $1 \mathrm{GHz} \beta$ is typically in the order of $1-10$ and $\phi$ between 0 and $-\pi / 2$. The case of $m=0$ can, of course, not be fully realized in practice, but is included in order to be able to compare with a pure FM spectrum.

Using (30) an approximate analytic expression for $S_{m}(f)$ can be found in the case of weak IM $(m \ll 1)[16]$

$$
\begin{aligned}
S_{m}(f)= & \sum_{n=-\infty}^{\infty}\left|J_{n}(\beta)-\frac{m}{4}\left(J_{n+1}(\beta) e^{j \phi}+J_{n-1}(\beta) e^{-j \phi}\right)\right|^{2} \\
& \cdot \delta\left(f-n f_{m}\right)
\end{aligned}
$$

with $\phi=\phi_{f}\left(I_{o}, f_{m}\right)$. For larger $m$ values a numerical calculation is necessary. We see that the pure FM spectrum $(m=0)$ is symmetric about $n=0$ (the carrier component), whereas the IM generally causes the spectrum to become asymmetric. This is a characteristic property for a combined IM and FM signal.

The phenomenon is further illustrated by the first series of spectra, Fig. 6(a)-(f). Here a $\beta$ value of $2.405[=$ first zero of $\left.J_{o}(x)\right]$ has been chosen and the three figures in the left column have a $\phi$ value of 0 , while those in the right column have $\phi$ equal to $-\pi / 2$. The three rows of figures have $m=0.0,0.5$, and 1.0 , respectively, taken from the top. All spectra are ob- 


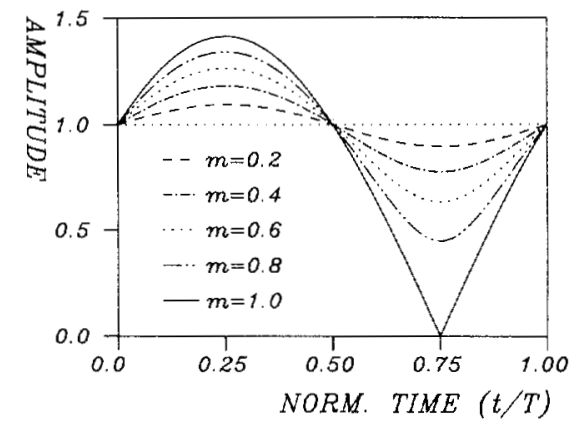

Fig. 2. Temporal variation over one period of the amplitude of the modulation function $m(t)$ (19) for sinusoidal modulation, with $m$ as parameter.

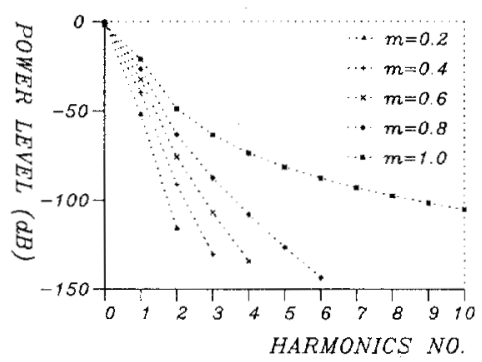

Fig. 3. Power spectrum of the square-root amplitude of $m(t)$, Fig. 2 , for sinusoidal intensity modulation, with $m$ as parameter.

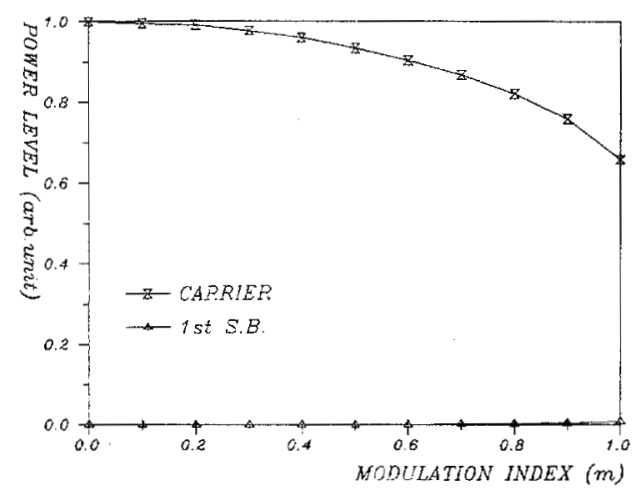

Fig. 4. Power level of carrier and first sideband versus IM index (linear scale). Pure intensity modulation.

tained numerically from $m(t)$ by a fast Fourier transform (FFT).

Some important tendencies can be deduced from these figures. For the pure FM spectrum $m=0$, the carrier component is 0 (31) regardless of $\phi=0$ or $-\pi / 2$. With increasing $m$ the spectrum becomes more and more skew for $\phi=0$ with a relative increase of the lower sidebands, while it remains symmetric for $\phi=-\pi / 2$. For $\phi=0$ the carrier component remains (close to) zero, while it increases significantly for $\phi=-\pi / 2$.

The degree of skewness of the spectrum is therefore dependent on the values of $\phi$ together with the IM index. If $\phi$ were equal to $-\pi$ the spectrum would be equally skew as for $\phi=0$, but with the upper sidebands enhanced instead of the lower ones. For a given $\phi$ value (which permits skewness) the degree of skewness is indicative of the amount of IM.

The next example is for $\beta=100$ and $\phi=0$, Fig. 7(a)-(c), corresponding to a value of $f_{m}$ less than approximately 10 MHz. Again the figures represent $m=0,0.5$, and 1.0, and the tendency of spectral skewness is the same as in the previous

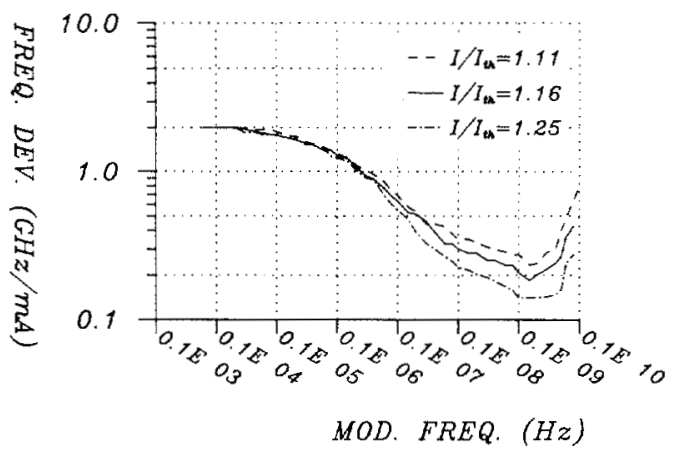

(a)

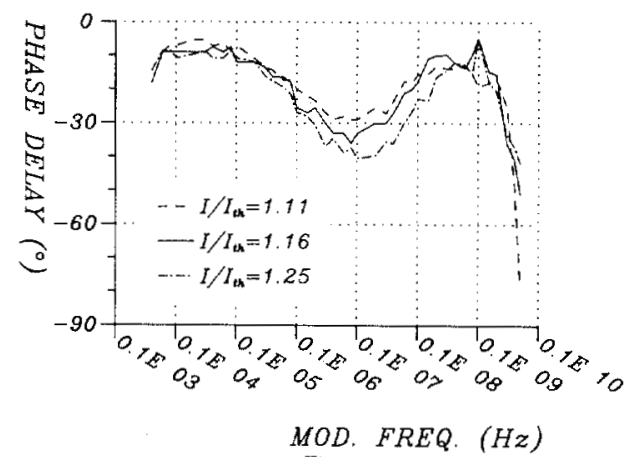

(b)

Fig. 5. Measured example of the current-to-frequency modulation transfer function for a CSP laser [16]. (a) Modulus. (b) Phase. Current values: $--: I_{o} / I_{\mathrm{th}}=1.11 ;-: I_{o} / I_{\mathrm{th}}=1.16 ; \cdots-: I_{o} / I_{\mathrm{th}}=$ 1.25 .

example. This is a broad-band FM case and we have therefore included the limiting distribution for large $\beta$ (28) given by

$$
S_{\mathrm{lim}}(n)=\frac{1-m \cdot n / \beta}{\pi \beta \sqrt{1-(n / \beta)^{2}}}
$$

where $n$ is the harmonics no. The amplitude of the harmonics is seen to assume almost random values in a region between zero and $2 \cdot S_{\lim }(n)$ (although there is a certain substructure). Therefore, it seems reasonable that the limit distribution may be obtained by averaging over a narrow frequency band (containing several sidebands), with increasing accuracy as $\beta \rightarrow \infty$ [20]. By asymptotic expansion of the Bessel functions (31) it can be shown that (32) is valid to including $O(m)$.

\section{B. Sawtooth Modulation}

Next, we will consider symmetric sawtooth modulation with $f(t)$ given as

$$
f(t)= \begin{cases}-1+4 \frac{t}{T}, & 0 \leqslant t \leqslant \frac{T}{2} \\ 3-4 \frac{t}{T}, & \frac{T}{2} \leqslant t \leqslant T .\end{cases}
$$

For simplicity we shall assume a flat frequency response without phase delay for $H_{f}$, so that $h_{f}\left(I_{o}, t\right)$ becomes

$$
h_{f}\left(I_{o}, t\right)=H_{f}\left(I_{o}, 0\right) \cdot \delta(t) \text {. }
$$

Using the procedure outlined in Section II-A we find the in- 


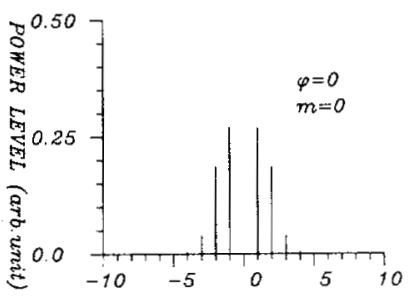

(a)

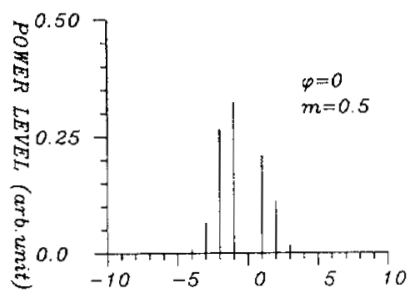

(b)

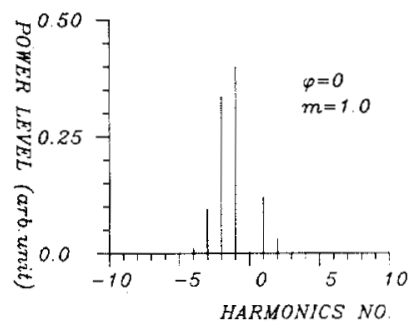

(c)

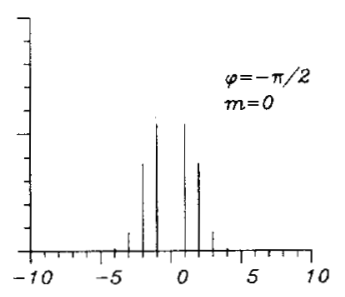

(d)

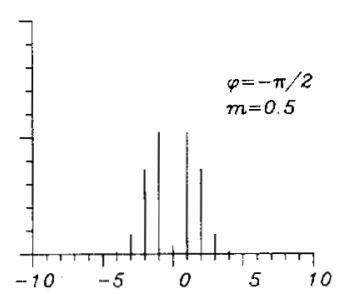

(e)

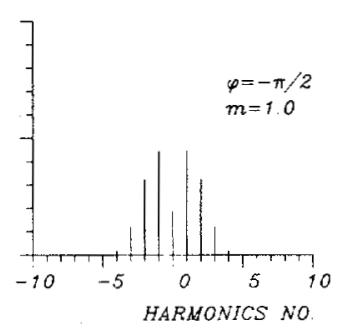

(f)
Fig. 6. Modulation power spectra for sinusoidal modulation with FM index $\beta=2.4$. (a) $\phi=0, m=0$. (b) $\phi=0, m=0.5$. (c) $\phi=0, m=$ 1.0. (d) $\phi=-\pi / 2, m=0$. (e) $\phi=-\pi / 2, m=0.5$. (f) $\phi=-\pi / 2, m=$ 1.0. ( $\phi$ is the phase delay $\phi_{f}\left(I_{O}, f_{m}\right)(19)$ and $m$ is the IM index.)

stantaneous frequency deviation

$$
\nu(t)= \begin{cases}-\Delta f\left(-1+4 \frac{t}{T}\right), & 0 \leqslant t \leqslant \frac{T}{2} \\ -\Delta f\left(3-4 \frac{t}{T}\right), & \frac{T}{2} \leqslant t \leqslant T\end{cases}
$$

where $\Delta f=I_{m} \cdot H_{f}\left(I_{o}, 0\right)$, showing a piecewise linear frequency sweep.

By integration of (35) the modulation function becomes

$$
S_{1 \mathrm{im}}(n)=\left(1-m \cdot \frac{n}{\beta}\right) \frac{1}{2 \beta} \text {. }
$$

It is shown in Fig. 8(a)-(c) as full lines.

\section{Square Wave Modulation}

This case is often considered for coherent applications and is known as frequency shift keying (FSK) modulation. Here $f(t)$ is given as

$$
f(t)=\left\{\begin{array}{rr}
-1, & 0 \leqslant t \leqslant \frac{T}{2} \\
1, & \frac{T}{2} \leqslant t \leqslant T
\end{array}\right.
$$

With $h_{f}$ given by $(34), \nu(t)$ becomes

$$
\nu(t)=\left\{\begin{array}{cc}
\Delta f, & 0 \leqslant t \leqslant \frac{T}{2} \\
-\Delta f, & \frac{T}{2} \leqslant t \leqslant T
\end{array}\right.
$$

where $\Delta f$ is defined as before.

The modulation function is then

$$
m(t)= \begin{cases}\sqrt{1-m} \exp \left\{j 2 \pi \beta\left(-\frac{1}{4}+\frac{t}{T}\right)\right\}, & 0 \leqslant t \leqslant \frac{T}{2} \\ \sqrt{1+m} \exp \left\{j 2 \pi \beta\left(\frac{3}{4}-\frac{t}{T}\right)\right\}, & \frac{T}{2} \leqslant t \leqslant T\end{cases}
$$

again with the FM index $\beta$ defined as usual.

In this case we expect the spectrum to be confined to narrow regions around $\pm \beta$. This is confirmed by the numerical examples. As in the earlier cases the skewness of the spectrum increases with increasing IM index. The final example to be presented here, Fig. 9(a)-(c), makes it possible to compare the three types of modulation we have considered. These figures show three pure FM spectra with $\beta=10$ and sinusoidal, sawtooth, and square wave modulation. The limit distribution for large $\beta$ is included in Fig. 9(a) and (b), while it consists of two

$$
m(t)= \begin{cases}\sqrt{1-m+4 m \frac{t}{T}} \exp \left\{-j 2 \pi \beta\left(-\frac{t}{T}+2\left(\frac{t}{T}\right)^{2}\right)\right\}, & 0 \leqslant t \leqslant \frac{T}{2} \\ \sqrt{1+3 m-4 m \frac{t}{T}} \exp \left\{-j 2 \pi \beta\left(3 \frac{t}{T}-2\left(\frac{t}{T}\right)^{2}-1\right)\right\}, & \frac{T}{2} \leqslant t \leqslant T .\end{cases}
$$

Even in this case we see that it is possible to define an FM index $\beta=\Delta f / f_{m}(=\Delta f T)$, where $\Delta f$ is the peak frequency deviation.

Numerical examples of the power spectrum associated with (36) are shown in Fig. 8(a)-(c). This is also a broad-band case with $\beta=100$, and we see, as expected, that the sidebands of highest level form a flat spectrum due to the linear frequency sweep of $\nu(t)$. With increasing IM there is the same skewness tendency as for sinusoidal modulation, and also the spectrum is confined to the region between $-\beta$ and $\beta$. The limit distribution $(28)$ is in this case given to including $O(m)$ as $\delta$ functions at $\pm \beta$ in Fig. $9(\mathrm{c})$. We see that the square wave spectrum is clearly concentrated at the edges while there are no significant differences between the sinusoidal and the sawtooth spectrum. Apparently, the two latter cases require a higher $\beta$ value to approach the limit distribution.

\section{Measurement Results}

This section presents measurement results of modulated power spectra for a CSP injection laser emitting at $832 \mathrm{~nm}$. The laser had a threshold current of $77 \mathrm{~mA}$. It was placed in 


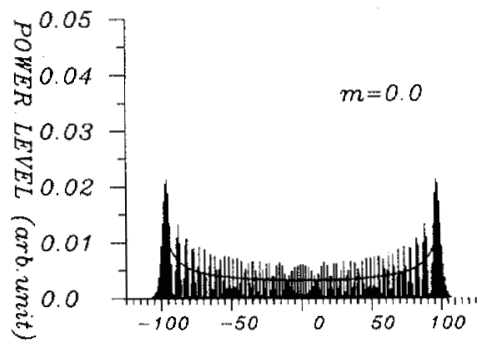

(a)

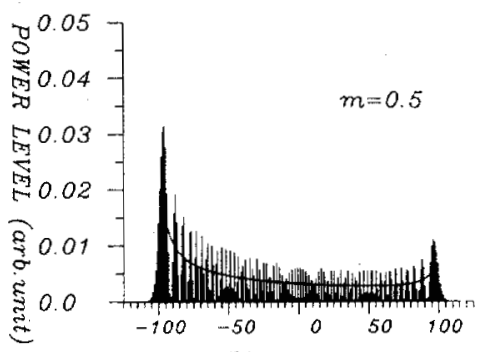

(b)

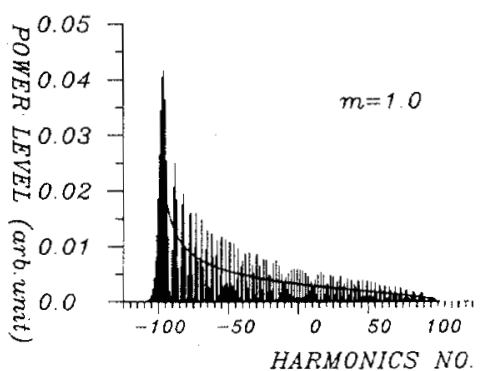

(c)

Fig. 7. Broad-band modulation power spectra for sinusoidal modulation $\beta=100, \phi=0$. (a) $m=0$. (b) $m=0.5$. (c) $m=1.0$. Limit distributions for large $\beta$ included as full lines.

an isolated box and mounted on a copper heat sink connected to a cooling/heating finn via a Peltier element. During the measurements the laser was temperature stabilized to within $0.01^{\circ} \mathrm{C}$ and kept at $24.00^{\circ} \mathrm{C}$. All measurements were taken using a scanning confocal mirror type Fabry-Perot interferometer (FPI). In the Appendix the connection between the spectrum displayed on the oscilloscope and the "true" spectrum is given.

The FPI had a free spectral range FSR $=2 \mathrm{GHz}$ and a finesse $F \simeq 150$ when used for measurements with low $F M$ index $(\beta<5)$. In this case the condition of $f_{m} \gg 2 \Delta f_{1 / 2}$ (see Appendix) allows a true oscilloscope display of the spectrum. For broad-band FM measurements $(\beta \gg 5)$ the FPI had FSR $=$ $7.5 \mathrm{GHz}$ and $F \simeq 100$. Here the conditions for a true display of the spectral envelope (A24), (A25) were fulfilled in all cases. The measurement examples comprise sinusoidal, sawtooth, and square wave modulation as presented in the previous section.

\section{A. Sinusoidal Modulation}

Our first example of Fig. 10 shows an $F M$ index $\beta \simeq 2.4$. The detailed data for the measurement are $f_{m}=150 \mathrm{MHz}$, $I_{o} / I_{\mathrm{th}}=1.21, m=0.24$. The spectrum displays first, second, and third order sidebands clearly and the amplitude of the lower sidebands is higher than that of the upper sidebands.

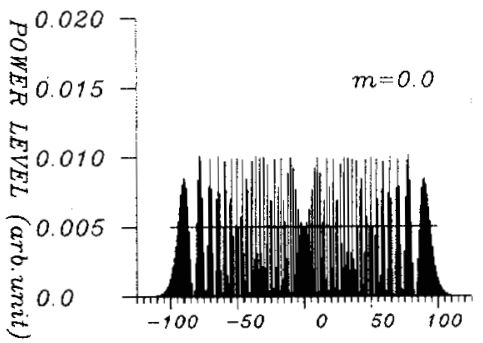

(a)

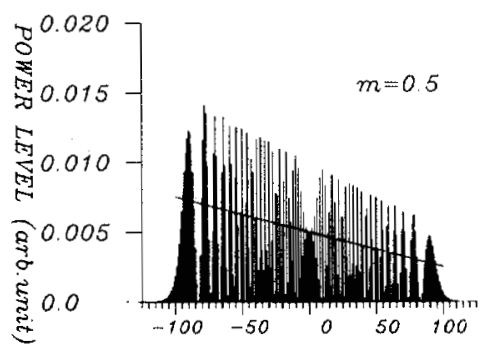

(b)

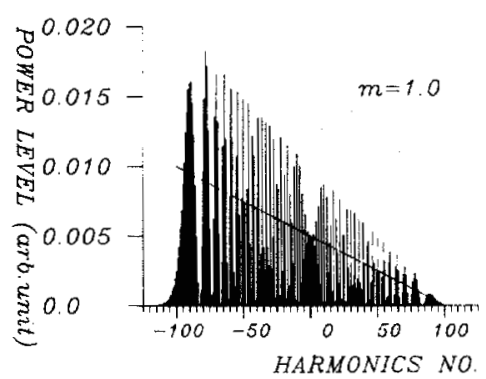

(c)

Fig. 8. Broad-band modulation power spectra for sawtooth modulation, $\beta=100$. (a) $m=0$. (b) $m=0.5$. (c) $m=1.0$. Limit distributions for large $\beta$ included as full lines.

From the skewness of the spectrum compared to Fig. 6 of the previous section, it appears that we have a $\phi$ value close to 0 . This is in qualitative agreement with the results for modulus and phase of the transfer function for a CSP laser $H_{f}\left(I_{o}, f\right)$ shown in Fig. 5(a), (b).

For a slightly higher value of $\beta, \beta \simeq 3.8$ (where the first order sidebands disappear in pure FM cases) we obtain Fig. 11 with the additional parameters $f_{m}=100 \mathrm{MHz}, I_{o} / I_{\text {th }}=1.22$, $m=0.28$. Again we have a skew spectrum $[\sim \phi \simeq 0$ in Fig. $5(b)]$ with clearly displayed peaks for the carrier and second, third, and fourth order sidebands where the lower sidebands have the largest magnitude.

Next, we consider a broad-band case with parameters $\beta \simeq$ $140, f_{m}=10 \mathrm{MHz}, I_{o} / I_{\text {th }}=1.17, m=0.50$ (Fig. 12). Here, we obtain from the FPI measurement only a display of the spectral envelope (32). There is a good qualitative agreement with the case considered in Fig. 7(b). For this figure and the measurement result we have (32) a ratio between the limiting distribution for $f=f_{o}-\Delta f$ and $f=f+\Delta f$ of $3: 1$.

As an example of broad-band modulation with a weak IM component we show Fig. 13 with $\beta \simeq 10^{5}, f_{m}=10 \mathrm{kHz}$, $I_{o} / I_{\text {th }}=1.16, m=0.05$. Here we have, as expected, $\phi \simeq 0$ and good qualitative agreement with Fig. 7(a) of the previous section with $m=0$. The skewness of the spectrum is less 


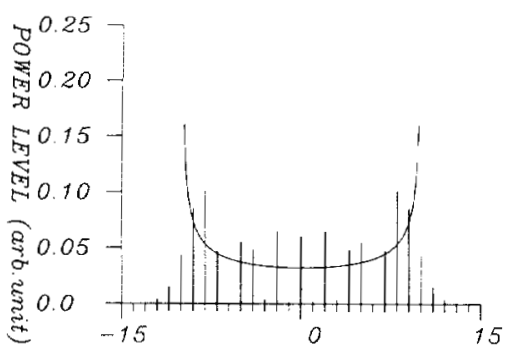

(a)

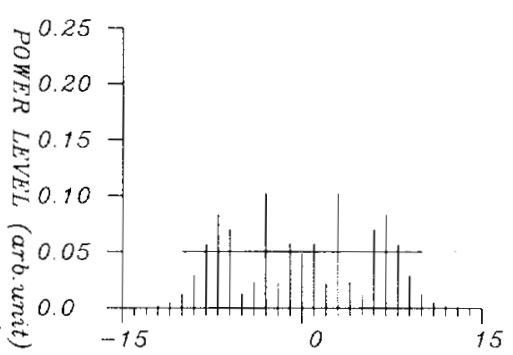

(b)

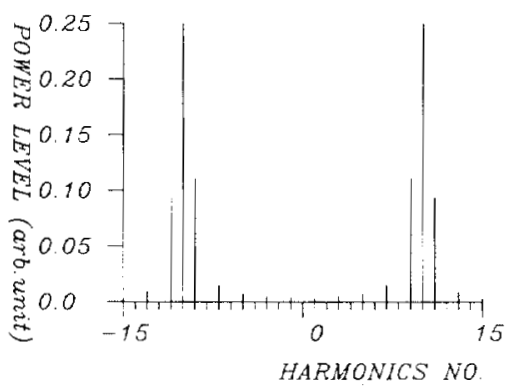

(c)

Fig. 9. Comparison of modulation power spectra with $\beta=10.0$ (pure FM). (a) Sinusoidal modulation. (b) Sawtooth modulation. (c) Square wave modulation. Limit distribution for large $\beta$ included as the full lines in (a) and (b) (for (c) the limit distribution consists of two $\delta$ functions).

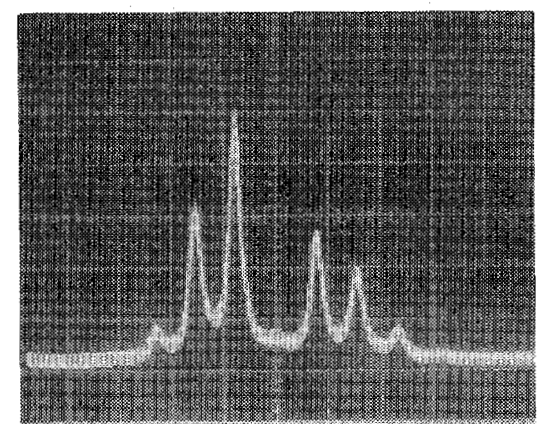

Fig. 10. Measured power spectrum for sinusoidal modulation $\beta \simeq 2.4$ showing almost complete extinction of the carrier. Experimental parameters: $I_{o} / I_{\mathrm{th}}=1.21, I_{m}=3.9 \mathrm{~mA} \mathrm{~A}_{o-p}, f_{m}=150 \mathrm{MHz}, m=0.24$. Horizontal axis: $200 \mathrm{MHz} /$ div.

pronounced than in the spectrum of Fig. 12. In this case the individual peaks displayed are connected with the scanning of the FPI (see the Appendix), and they cannot be directly related to spectral sidebands. However, the envelope of the figure is in agreement with the spectral envelope since (A24) and (A25) are fulfilled.

\section{B. Sawtooth Modulation}

An example of a broad-band sawtooth modulation spectrum is shown in Fig. $14\left(\beta \simeq 1.9 \times 10^{5}, f_{m}=8 \mathrm{kHz}, I_{o} / I_{\mathrm{th}}=1.17\right.$,

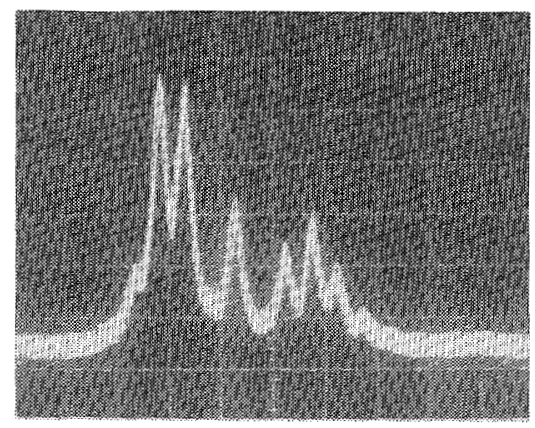

Fig. 11. Measured power spectrum for sinusoidal modulation $\beta \simeq 3.8$. Experimental parameters: $I_{o} / I_{\text {th }}=1.22, I_{m}=4.8 \mathrm{~mA}_{o-p}, f_{m}=100$ $\mathrm{MHz}, m=0.28$. Horizontal axis: $200 \mathrm{MHz} /$ div.

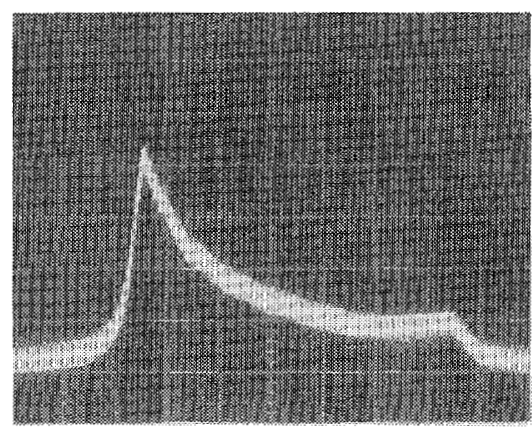

Fig. 12. Measured broad-band power spectrum for sinusoidal modulation, $\beta \simeq 140$. Only a spectral envelope is displayed. Experimental parameters: $I_{o} / I_{\mathrm{th}}=1.17, I_{m}=6.5 \mathrm{~mA}_{o-p}, f_{m}=10 \mathrm{MHz}, m=0.50$. Horizontal axis: $400 \mathrm{MHz} /$ div.

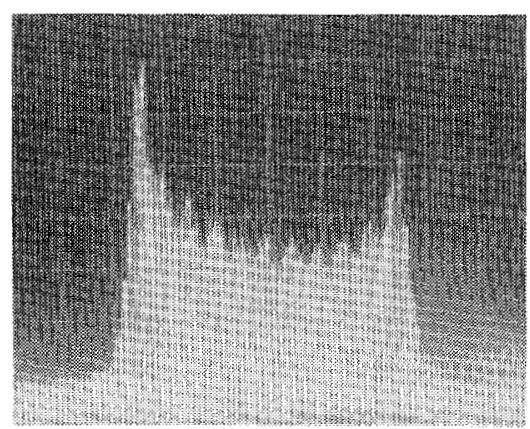

Fig. 13. Measured broad-band power spectrum for sinusoidal modulation $\beta \simeq 10^{5}$. Separate peaks are displayed for each crossing of the instantaneous laser frequency through the FPI passband. Experimental parameters: $I_{o} / I_{\mathrm{th}}=1.16, I_{m}=0.60 \mathrm{~mA}_{o-p}, f_{m}=10 \mathrm{kHz}, m=$ 0.05 . Horizontal axis: $400 \mathrm{MHz} /$ div.

$m=0.07$ ). Due to the low IM component the spectrum has only a slight skewness and an almost equal distribution between $f_{o}-\Delta f$ and $f_{o}+\Delta f$ in agreement with the calculated results of Section III-B [Fig. 8(a)]

\section{Square Wave Modulation}

We have exemplified square wave modulation by choosing in Fig. 15 the parameters $\beta \simeq 6.3 \times 10^{4}, f_{m}=30 \mathrm{kHz}, I_{o} / I_{\text {th }}=$ 1.17, $m=0.07$. We have, as expected from Fig. 9(c) in Section III-C, an almost symmetrical spectral distribution with a concentration of significant spectral components near the edges of the spectrum. Deviations from the "ideal" spectrum of Fig. 9(c) may be attributed to the finite rise- and fall-times of $\nu(t)$ (39) and the nonuniform frequency response of $H_{f}$. 


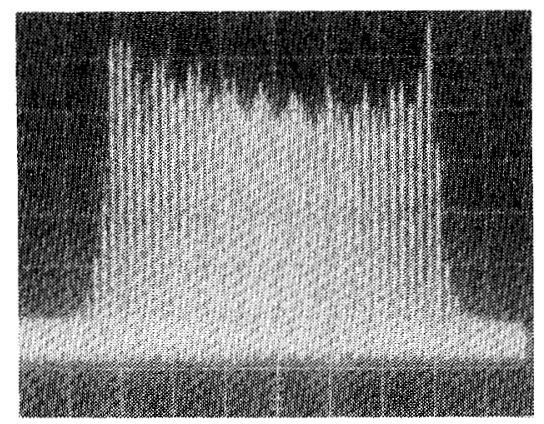

Fig. 14. Measured broad-band power spectrum for sawtooth modulation $\beta \simeq 1.9 \cdot 10^{5}$. Individual peaks are connected with different passages as for Fig. 13. The peak envelope is almost flat over a wide range indicating the more linear frequency sweep than in the case of Fig. 13. Experimental parameters: $I_{o} / I_{\mathrm{th}}=1.17, I_{m}=0.86 \mathrm{~mA}_{o-p}$, $f_{m}=8 \mathrm{kHz}, m=0.07$. Horizontal axis: $500 \mathrm{MHz} /$ div.

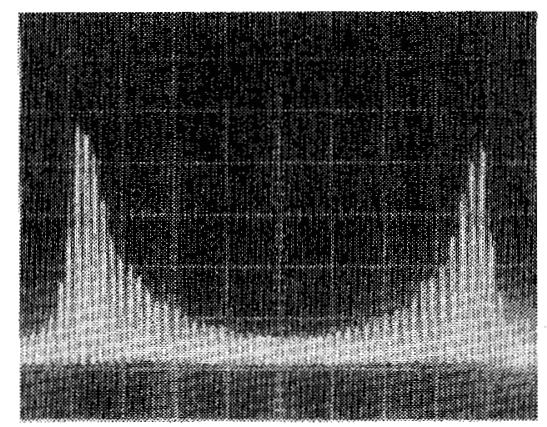

Fig. 15. Measured broad-band power spectrum for square wave modulation (FSK) $\beta \simeq 6.3 \cdot 10^{4}$. Individual peaks are connected with different passages as for Fig. 13. The spectrum is concentrated in the regions near $f_{o} \pm \Delta f$. Experimental parameters: $I_{o} / I_{\text {th }}=1.17, I_{m}=$ $0.9 \mathrm{~mA}_{o-p}, f_{m}=30 \mathrm{kHz}, m=0.07$. Horizontal axis: $500 \mathrm{MHz} /$ div.

\section{CONCLUSION}

This paper has presented a detailed theoretical and experimental investigation of the spectral behavior of a current modulated injection laser.

In the theoretical analysis a general formulation is presented for the laser field obtained from a combined intensity and frequency modulation (IM + FM). The theory is based on the knowledge of the current-to-frequency modulation transfer function (CF-MTF) for the laser. The general formulas for the resulting power spectrum show that it is obtained from a translation of the spectrum for the modulation function to the center frequency of the laser followed by a convolution with the spectral line shape of the unmodulated laser.

Numerical examples have been presented dealing with sinusoidal, sawtooth, and square wave (FSK) modulation. From the results it is obvious that the pure FM spectral shape is strongly influenced by the IM index and the phase delay between the IM and the FM part of the modulating signal. In the case of zero phase delay the spectrum becomes more and more asymmetric (skew) for increasing $\mathbf{M}$ index with a relative increase of the lower sidebands. For a phase delay between 0 and $-\pi / 2$ a similar conclusion is drawn although the skewness tendency is less pronounced for decreasing phase delay values. For a phase delay of $-\pi / 2$ the spectrum remains symmetrical for increasing IM index, whereas the relative sideband magnitude changes significantly from the pure FM case. When the phase delay lies between $-\pi / 2$ and $-\pi$ we obtain a reverse situation to the one for a delay between 0 and $-\pi / 2$, resulting in a relative increase of higher sidebands.

Measurements of the power spectrum for a temperature stabilized CSP injection laser were taken using a Fabry-Perot interferometer (FPI). In such measurements a detailed understanding of the characteristics of the FPI and the detection system is essential in order to interpret the results correctly. A thorough consideration of this problem has been given and is outlined in the Appendix. Taking the characteristics into account the measurements have confirmed qualitatively our calculation results, where we have considered broad-band and narrow-band FM cases for sinusoidal modulation as well as broad-band cases for sawtooth and square wave modulation. The results are in agreement with an earlier measurement of modulus and phase of the CF-MTF for this type of injection laser [16]. The present investigation may serve as an important tool for the understanding of the behavior of currentmodulated injection lasers when applied in coherent or heterodyne communication systems [3], [6], [7], [16]. Also, it could be of importance when considering in detail the effect of using such a laser injection locked to another laser, whereby a reduction of the IM appears [8], or phase modulation (PM) occurs [21].

The main limitations of the presented model are the need for knowledge of the complete complex CF-MTF of the laser and the neglect of transient IM effects, causing problems at high modulation frequencies $\left(f_{m} \gtrsim 500 \mathrm{MHz}\right)$. The modulus of the CF-MTF can be measured up to several $\mathrm{GHz}$ and can also be predicted from a rate equation analysis [6]. So far, the phase delay has only been measured up to approximately $500 \mathrm{MHz}$. In future work it could be analyzed if the phase delay can also be obtained from the rate equations or from the information contained in the modulus. Also, it could be of interest to extend the measurement of the phase delay to higher frequencies. Transient IM effects might be incorporated in the present theory by introducing a transfer function from current to intensity modulation.

\section{APPENDIX \\ Measurement of Modulated Laser Spectra WITH A FABRY-PERot INTERFEROMETER (FPI)}

Spectral measurements with an FPI are often modified by the characteristics of the interferometer and the detection system. A thorough understanding of this influence is necessary for a correct interpretation of the obtained results.

The FPI will in most cases be a scanning confocal spherical mirror type [22]. The mirrors are coated with highly reflecting dielectric material and the incoming light beam is therefore reflected several times inside the cavity. If the wavelength of the light matches the cavity length, constructive interference will take place and an amplified light beam is transmitted through the FPI to the detector.

The intensity transfer function of the FPI may be written [22]

$$
H_{\mathrm{FPI}}(f)=\frac{1}{1+(2 F / \pi)^{2} \sin ^{2}[\pi(f / \mathrm{FSR})]}
$$

where $F$ and FSR are the finesse and the free spectral range of the FPI, respectively. The resolution of the FPI is then given as 


$$
2 \Delta f_{1 / 2}=\frac{\text { FSR }}{F} .
$$

The finesse is limited by the mirror reflectivity, surface roughness of the mirrors, diffraction losses, pinhole size, etc. [23]. For simplicity, we will define an effective mirror reflectivity $R$ so that

$$
F=\frac{\pi \sqrt{R}}{1-R} .
$$

This allows us to write the field amplitude transfer function as

$$
A_{\mathrm{FPI}}(f)=(1-R) \sum_{k=0}^{\infty} R^{k} e^{-j 2 \pi f k \tau}
$$

where $\tau$ is equal to $1 / \mathrm{FSR}$. The relation between $H_{\mathrm{FPI}}(f)$ and $A_{\text {FPI }}(f)$ is given by

$$
H_{\mathrm{FPI}}(f)=\left|A_{\mathrm{FPI}}(f)\right|^{2} \text {. }
$$

The impulse response corresponding to (A4) is

$$
a_{\mathrm{FPI}}(t)=(1-R) \sum_{k=0}^{\infty} R^{k} \delta(t-k \tau) .
$$

With the input field given by (4), the output field from the FPI becomes

$$
E_{\text {out }}(t)=(1-R) \sum_{k=0}^{\infty} R^{k} E(t-k \tau)
$$

In the following we shall calculate the FPI spectrum, as it will be displayed on an oscilloscope, in two limiting cases.

\section{A. The High-Frequency Region $f_{m} \gg 2 \Delta f_{1 / 2}$}

For $m(t)$ we use the Fourier expansion (22). In practice the oscilloscope display and the multiple FPI scans give rise to an ensemble-averaged intensity

$$
I_{d}(t)=\left\langle\left|E_{\text {out }}\right|^{2}\right\rangle \text {. }
$$

Using (22), (4), and (A7) we find

$$
\begin{aligned}
I_{d}(t)= & \langle|(1-R) \sum_{k=0}^{\infty} R^{k} \sum_{n=-\infty}^{\infty} \gamma_{n} r(\tau-k \tau) \\
& \left.\left.\cdot e^{j 2 \pi\left(f_{o}+n f_{m}\right)(t-k \tau)|2\rangle}\right|^{2}\right\rangle \\
= & \sum_{n=-\infty}^{\infty} \sum_{m=-\infty}^{\infty} \gamma_{n} \gamma_{m}^{*} e^{j 2 \pi(n-m) f_{m} t} \\
& \cdot(1-R)^{2} \sum_{k=0}^{\infty} \sum_{l=0}^{\infty} R^{k+1}\left\langle r(t-k \tau) r^{*}(t-l \tau)\right\rangle \\
& \cdot e^{-j 2 \pi(k-t) f_{O} \tau} e^{-j 2 \pi(k n-l m) f_{m} \tau} .
\end{aligned}
$$

As $r(t)$ is assumed to be a stationary process we get

$$
\left\langle r(t-k \tau) r^{*}(t-l \tau)\right\rangle=R_{o}((k-l) \tau)
$$

where we have also assumed that $S_{o}(f)$ is an even function of $f$, so that $R_{o}(\tau)$ is a real and even function of $\tau$.
Next, we introduce the detection bandwidth $B$ and we will assume that $B \ll f_{m}$. In this case $I_{d}(t)$ will be time-averaged over several periods and we find

$$
\frac{1}{T_{d}} \int_{t-T_{d}}^{t} e^{j 2 \pi(n-m) f_{m} t} d t \simeq \delta_{n m}
$$

where $T_{d}=1 / B$ and $\delta_{n m}$ is the Kronecker delta symbol.

The expression (A9) then simplifies to

$$
\begin{aligned}
\overline{I_{d}(t)}= & \sum_{n=-\infty}^{\infty}\left|\gamma_{n}\right|^{2}(1-R)^{2} \\
& \cdot \sum_{k=0}^{\infty} \sum_{l=0}^{\infty} R^{k+l} e^{-j 2 \pi\left(f_{O}+n f_{m}\right)(k-l) \tau} R_{o}((k-l) \tau) .
\end{aligned}
$$

Using (3) we obtain

$$
\begin{aligned}
\overline{I_{d}(t)} & =\sum_{n=-\infty}^{\infty}\left|\gamma_{n}\right|^{2} \\
& \cdot \int_{-\infty}^{\infty} S_{o}(f) \cdot\left|(1-R) \sum_{k=0}^{\infty} R^{k} e^{j 2 \pi\left(f-f_{o}-n f_{m}\right) k \tau}\right|^{2} d f \\
& =\sum_{n=-\infty}^{\infty}\left|\gamma_{n}\right|^{2} \cdot\left[S_{o}(f) \oplus H_{\mathrm{FPI}}(f)\right]_{f=f_{o}+n f_{m}} \\
& =\sum_{n=-\infty}^{\infty}\left|\gamma_{n}\right|^{2} \cdot H_{\mathrm{FPI}}^{\prime}\left(f_{o}+n f_{m}\right)
\end{aligned}
$$

where $H_{\text {FPI }}^{\prime}(f)$ is a modified transfer function for the FPI which is the original one (A1) convolved with the spectral line shape $S_{o}(f)$.

Until now the scanning of the FPI cavity length has been neglected. During this scan a particular transmission peak of $H_{\text {FPI }}^{\prime}(f)$ will sweep across the spectrum $S_{m}(f)$. With the assumption $f_{m} \gg 2 \Delta f_{1 / 2}$ only one sideband at a time will fall within the peak. Each spectral component is therefore displayed separately with the correct amplitude relative to the others and a shape given by $H_{\mathrm{FPI}}^{\prime}(f)$ (Figs. 10 and 11). For lower modulation frequencies the sidebands will start overlapping and a spectral "envelope" will be displayed (Fig. 12).

\section{B. The Low Frequency Region $f_{m} \ll 2 \Delta f_{1 / 2}$}

In this case the basic assumption is that the modulation function $m(t)$ can be regarded as a pure sine wave with "frozen" amplitude and frequency during the measurement time of the FPI $\left(T_{\mathrm{FPI}} \simeq 1 /\left(2 \Delta f_{1 / 2}\right)\right)$. We therefore write

$$
m(t) \simeq\left|m\left(t\left(v_{d}\right)\right)\right| e^{j 2 \pi v_{d}(t) \cdot t}
$$

where $v_{d}(\mathrm{t})$ is the instantaneous frequency deviation (13) and $\left|m\left(t\left(v_{d}\right)\right)\right|$ is the amplitude of $m(t)$ at the time where the frequency deviation equals $v_{d}$.

The approximate field expression becomes

$$
E(t) \simeq\left|m\left(t\left(v_{d}\right)\right)\right| r(t) e^{j 2 \pi\left(f_{o}+v_{d}(t)\right) t} .
$$


Proceeding as before we find the FPI output intensity

$$
\begin{aligned}
I_{d}(t)= & \left\langle\left|E(t) \oplus a_{\mathrm{FPI}}(t)\right|^{2}\right\rangle \\
\simeq & \left|m\left(t\left(\nu_{d}\right)\right)\right|^{2} \cdot(1-R)^{2} \\
& \cdot \sum_{k=0}^{\infty} \sum_{l=0}^{\infty} R^{k+l}\left\langle r(t-k \tau) r^{*}(t-l \tau)\right\rangle \\
& \cdot e^{-j 2 \pi\left(f_{o}+v_{d}(t)\right)(k-l) \tau} \\
= & \left|m\left(t\left(\nu_{d}\right)\right)\right|^{2} \cdot\left[S_{o}(f) \oplus H_{\mathrm{FPI}}(f)\right]_{f=f_{o}+v_{d}(t)} \\
= & \left|m\left(t\left(v_{d}\right)\right)\right|^{2} \cdot H_{\mathrm{FPI}}^{\prime}\left(f_{o}+\nu_{d}(t)\right) .
\end{aligned}
$$

The spectrum, which is displayed on the oscilloscope, will only under certain conditions resemble the theoretical limit distribution of (28). The actual picture is determined by

1) the scanning speed of the FPI, $d v_{\mathrm{FPI}} / d t$,

2) the derivative of the laser frequency $\left|d \nu_{d}\right| d t \mid$, and

3) the detection bandwidth $B$.

If the laser frequency $f_{o}+v_{d}$ were constant, a peak with the shape and amplitude given by (A16) would be displayed during the scan of the FPI. The combined effect of the FPI scanning and the laser frequency change is a "pulse compression" of the transmission peak with the factor

$$
K=1+\frac{d \nu_{d} / d t}{d \nu_{\mathrm{FPI}} / d t}
$$

However, for small values of $d v_{d} / d t$, where the rise time of the pulse is greater than the detector time constant $T_{d}$ (A11) the peak amplitude is independent of $d v_{d} / d t$. It should be noted that the peaks are no longer associated with the sidebands of the true spectrum.

For higher modulation frequencies and/or peak frequency deviations, the response of the detection system becomes important and the detected signal is found by convolution

$$
\begin{aligned}
i_{d}(t) & =I_{d}(t) \oplus h_{d}(t) \\
& \simeq\left|m\left(t\left(v_{d}\right)\right)\right|^{2} \cdot\left[H_{\mathrm{FPI}}^{\prime}\left(f_{o}+v_{d}(t)\right) \oplus h_{d}(t)\right] .
\end{aligned}
$$

For simplicity the impulse response $h_{d}(t)$ of the detection system is taken to be

$$
h_{d}(t)= \begin{cases}\frac{1}{T_{d}}, & 0<t<T_{d} \\ 0, & \text { elsewhere. }\end{cases}
$$

The convolution in (A18) then yields

$$
\begin{aligned}
i_{d}(t) & =\left|m\left(t\left(\nu_{d}\right)\right)\right|^{2} \cdot \frac{1}{T_{d}} \int_{0}^{T_{d}} H_{\mathrm{FPI}}^{\prime}\left(f_{o}+v_{d}\left(t-t^{\prime}\right)\right) d t^{\prime} \\
& \simeq\left|m\left(t\left(\nu_{d}\right)\right)\right|^{2} \cdot \frac{1}{B^{\prime}} \int_{0}^{B^{\prime}} H_{\mathrm{FPI}}^{\prime}\left(f_{o}+\nu_{d}(t)-f^{\prime}\right) d f^{\prime}
\end{aligned}
$$

where $f^{\prime}=d v_{d} / d t \cdot t^{\prime}$ and $B^{\prime}=d \nu_{d} / d t \cdot T_{d}$. If $B^{\prime} \gg 2 \Delta f_{1 / 2}$, the pulse amplitude of $i_{d}(t)$ will be inversely proportional to
$B^{\prime}$, i.e., to the time interval in which the instantaneous frequency is in the FPI transmission band [14].

From (A20) the connection to the probability distribution of the instantaneous frequency is established. The probability that the instantaneous frequency falls within a narrow band of width $\delta \nu$ around $f_{o}+v_{d}$ is equal to the fraction of time in which this occurs. All frequency values between the extrema will be passed (at least) twice during one period so the probability density becomes

$$
w(f)= \begin{cases}\frac{1}{T} \cdot \sum\left\{\left|\frac{d \nu_{d}}{d t}\right|_{\nu_{d}=f}\right\}^{-1}, & |f|<\Delta f \\ 0, & \text { elsewhere. }\end{cases}
$$

Here $T$ is the modulation period and the summation covers the number of passages of $f$ during one period of $m(t)$.

In the case of a significant IM content in the modulation, combined with a phase delay different from zero, the intensity will be different for the different passages. The contributions to the sum in (A21) are therefore weighted with the corresponding intensity $\left|m\left(t\left(v_{d}\right)\right)\right|^{2}$ so that the following limit distribution arises

$$
S_{\lim }(f)= \begin{cases}\frac{1}{T} \cdot \sum\left\{\frac{\left|m\left(t\left(\nu_{d}\right)\right)\right|^{2}}{\left|\frac{d v_{d}}{d t}\right|}\right\}_{v_{d}=f} & |f|<\Delta f \\ 0, & \text { elsewhere. }\end{cases}
$$

For the FM index $\beta$ approaching infinity this distribution corresponds to the average of the true spectrum $S_{m}(f)$ over a narrow frequency band (which still contains several sidebands) [20]. Only in the case of equal intensity for each passage (A22) can be simplified to

$$
S_{\lim }(f)=|m(t(f))|^{2} \cdot w(f) .
$$

Experimentally, it will often be possible to distinguish the two contributions to the sum in (A22) in the FPI spectrum. According to (A20) the associated peaks are displayed separately, as they do not occur simultaneously. An example of the time variations of $\nu_{d}(t)$ and $\nu_{\mathrm{FPI}}(t)$ and crossings are shown in [14], Fig. 2 for the case of square wave modulation.

The conditions to be fulfilled for obtaining a correct display of the broad-band spectral envelope may now be stated as

$$
f_{m} \gg \frac{1}{T_{\text {scan }}}
$$

and

$$
\frac{2 \Delta f_{1 / 2}}{\left|d v_{d} / d t\right|} \ll T_{d}
$$

$T_{\text {scan }}$ is the ramp duration of the FPI.

The first condition (A24) assures that the instantaneous laser frequency will cross the FPI passband several times during one scan, while the second one (A25) assures that the time spent by the instantaneous frequency in the FPI passband is much less than the detection time constant. 
If $f_{m}$ is not too high and (A25) is valid, the FPI will display separate peaks for each passage of $\nu_{d}(t)$ with a height given by (A20) (Figs. 13-15). The peak envelope will then resemble $S_{\text {lim }}(f)$ (A23) in two cases, either 1 ) if one of the passages in a period has a small derivative $\left|d v_{d} / d t\right|$ compared to the others, or 2) if all passages have derivatives of nearly equal magnitude. Near the edges of the spectrum, where the direction of frequency change is reversed, (A25) may not be valid. At higher modulation frequencies the peaks will start overlapping each other, which gives an averaging similar to (A22) and improves the agreement with this expression. Eventually only the peak envelope will be displayed (Fig. 12).

The foregoing analysis has clarified the relation between the experimental and theoretical spectrum. The conditions(A24)(A25) may always be checked in practical cases to assure a correct measurement. Even if these conditions are not satisfied the width of the spectrum may still be correctly determined.

\section{ACKNOWLEDGMENT}

The authors wish to thank B. Tromberg of the Electromagnetics Institute for useful suggestions and discussions throughout this work.

\section{REFERENCES}

[1] Y. Yamamoto, "Receiver performance evaluation of various digital optical modulation-demodulation systems in the $0.5-$ $10 \mu \mathrm{m}$ wavelength region," IEEE $J$. Quantum Electron., vol. QE-16, pp. 1251-1260, Nov. 1980.

[2] Y. Yamamoto and T. Kimura, "Coherent optical fiber transmission systems," IEEE J. Quantum Electron., vol. QE-17, pp. 919-935, June 1981.

[3] S. Saito, Y. Yamamoto, and T. Kimura, "Optical FSK heterodyne detection experiments using semiconductor laser transmitter and local oscillator," IEEE J. Quantum Electron., vol. QE-17, pp. 935-941, June 1981.

[4] M. Nakamura, K. Aiki, N. Chinone, R. Ito, and J. Umeda, "Longitudinal-mode behaviors of mode-stabilized $\mathrm{Al}_{x} \mathrm{Ga}_{1-x} \mathrm{As}$ injection lasers," J. Appl. Phys., vol. 49, pp. 4644-4648, Sept. 1978.

[5] J. M. Osterwalder and B. J. Rickett, "Frequency modulation of GaAlAs injection lasers at microwave frequency rates," IEEE $J$. Quantum Electron., vol. QE-16, pp. 250-252, Mar. 1980.

[6] S. Kobayashi, Y. Yamamoto, and T. Kimura, "Modulation frequency characteristics of directly optical frequency modulated AlGaAs semiconductor laser," Electron. Lett., vol. 17, pp. 350351, May 1981.

[7] D. W. Smith and D. J. Malyon, "Experimental $1.51 \mu \mathrm{m}$ monomode fiber link containing an injection locked repeater," Electron. Lett. , vol. 18, pp. 43-45, Jan. 1982.

[8] S. Kobayashi, Y. Yamamoto, and T. Kimura, "Optical FM signal amplification and FM noise reduction in an injection locked AlGaAs semiconductor laser," Electron. Lett., vol. 17, pp. 849851, Oct. 1981.

[9] R. E. Epworth, "The phenomenon of modal noise in analogue and digital optical fiber systems," in Proc. 4th ECOC, Genova, Italy, Sept. 1978, pp. 492-501.

[10] H. Olesen, E. Nicolaisen, and M. Danielsen, "Quantitative experimental results on modal distortion and comparison with theory based on AM-FM conversion," in Proc. 6th ECOC, York, England, Sept. 1980 , pp. 84-87.

[11] R. E. Epworth and M. J. Pettitt, "Polarization modal noise and fiber birefringence in single mode fiber systems," in Proc. 3rd IOOC, San Francisco, CA, Apr. 1981, pp. 58-60.

[12] $\mathrm{H}$. Olesen and E. Nicolaisen, "Source wavelength instability and its consequence for dispersion measurements and interference noise in optical fibers," in Proc. 7th ECOC, Copenhagen, Denmark, Sept. 1981, pp. 4.1-1-4.1-4.
[13] M. Ito and T. Kimura, "Stationary and transient properties of semiconductor laser diodes," IEEE J. Quantum Electron., vol. QE-17,pp. 785-795, May 1981.

[14] L. Goldberg, H. F. Taylor, and J. F. Weller, "Time-dependent thermal effects in current-modulated semiconductor lasers," Electron. Lett., vol. 17, pp. 497-499, July 1981.

[15] M. Ito and T. Kimura, "Carrier density dependence of refractive index in AlGaAs semiconductor lasers," IEEE J. Quantum Electron., vol. QE-16, pp. 910-911, Sept. 1980.

[16] H. Olesen and G. Jacobsen, "Phase delay between intensity and frequency modulation of a semiconductor laser (including a new measurement method)," in Proc. 8th ECOC, Cannes, France, Sept. 1982, pp. 291-295.

[17] F. Kapron, "Baseband response function of monomode fibers," presented at the Topical Meet. on Optical Commun., Washington, DC, 1979, paper ThC5

[18] S. Saito, O. Nilsson, and Y. Yamamoto, "Oscillation center frequency tuning, Quantum FM noise, and direct frequency modulation characteristics in external grating loaded semicondcutor lasers," IEEE J. Quantum Electron., vol. QE-18, pp. 961-970, June 1982.

[19] S. O. Rice, "Mathematical analysis of random noise," Bell Syst. Tech. J., vol. 23, pp. 282-332, 1944; and Bell Syst. Tech. J., vol. 24, pp. $46-156,1945$.

[20] H. E. Rowe, Signals and Noise in Communication Systems. Princeton, NJ: Van Nostrand, 1965, pp. 120-123.

[21] S. Kobayashi and T. Kimura, "Optical phase modulation in an injection locked AlGaAs semiconductor laser," Electron. Lett., vol. 18 , pp. 210-211, Mar. 1982.

[22] M. Hercher, "The spherical mirror Fabry-Perot interferometer," Appl. Opt., vol. 7, pp. 951-966, May 1968.

[23] T. Takakura, K. Iga, and T. Tako, "Linewidth measurement of a single longitudinal mode AlGaAs laser with a Fabry-Perot interferometer," Japan. J. Appl. Phys., vol. 19, pp. L725-L727, Dec. 1980.

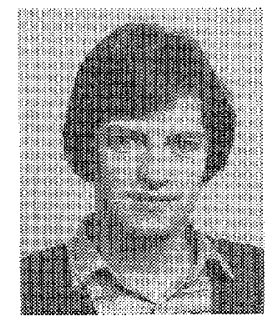

Henning Olesen was born in Vinderup, Denmark, on October 11,1955 . He received the M.Sc. degree in electrical engineering in 1980 from the Technical University of Denmark, Lyngby, Denmark.

Since 1980 he has been with the Electromagnetics Institute, Technical University of Denmark, where he is working towards the Ph.D. degree in optical communication. His research is primarily within the fields of semiconductor laser coherence, optical frequency modulation, and interference phenomena.

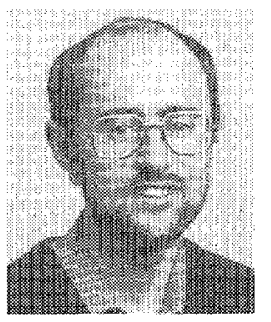

Gunnar Jacobsen was born in Copenhagen, Denmark, on April 14, 1952. He received the M.Sc. degree in electrical engineering and the Ph.D. degree from the Technical University of Denmark, Lyngby, Denmark, in 1977 and 1981, respectively.

His doctoral thesis work was conducted in the area of asymptotic description of propagation in refraction index ducts, with multimode graded index optical fibers as the most specific application. One year of the Ph.D. research period was spent at the Department of Electrical Engineering Polytechnic Institute of New York, Farmingdale, NY. From 1979 to 1981 he worked as an Assistant Professor at the Electromagnetics Institute, Technical University of Denmark. Since 1981 he has been engaged as a Research Fellow on coherent/heterodyne optical fiber communications systems.

Dr. Jacobsen is a member of the Optical Society of America. 